\title{
Disease Behaviour During the Peri-Diagnostic Period in Patients with Suspected Interstitial Lung Disease: The STARLINER Study
}

\author{
Marlies S. Wijsenbeek · Elisabeth Bendstrup • Claudia Valenzuela • Michael T. Henry • \\ Catharina C. Moor · Stéphane Jouneau • Alessandro G. Fois · Onofre Moran-Mendoza • \\ Syed Anees · Mirela Mirt · Monica Bengus · Frank Gilberg • Klaus-Uwe Kirchgaessler • \\ Carlo Vancheri
}

Received: April 9, 2021 / Accepted: May 13, 2021 / Published online: June 11, 2021

(C) The Author(s) 2021

\section{ABSTRACT}

Introduction: Disease behaviour may guide diagnosis and treatment decisions in patients with interstitial lung disease (ILD). STARLINER aimed to characterise disease behaviour in patients with suspected ILD during the peridiagnostic period using real-time home-based

Supplementary Information The online version contains supplementary material available at https:// doi.org/10.1007/s12325-021-01790-y.

M. S. Wijsenbeek $(\varangle) \cdot$ C. C. Moor

Department of Respiratory Medicine, Centre for Interstitial Lung Diseases and Sarcoidosis, Erasmus MC, University Medical Centre Rotterdam, 3015 GD Rotterdam, The Netherlands

e-mail: m.wijsenbeek-lourens@erasmusmc.nl

\section{E. Bendstrup}

Department of Respiratory Diseases and Allergy,

Centre for Rare Lung Diseases, Aarhus University

Hospital, Palle Juul-Jensens Boulevard 99,

8200 Aarhus N, Aarhus, Denmark

\section{Valenzuela}

Pulmonology Department, Instituto de

Investigación, Hospital Universitario de La Princesa,

Universidad Autonoma de Madrid, Calle de Diego

de León 62, 28006 Madrid, Spain

M. T. Henry

Department of Respiratory Medicine, Cork

University Hospital, Bishopstown Road,

Wilton T12 DFK4, Cork, Ireland assessments.

Methods: STARLINER (NCT03261037) was an international, multicentre study. Patients $\geq 50$ years old with suspected ILD were followed throughout the peri-diagnostic period, consisting of a pre-diagnostic period (from enrolment to diagnosis) and a post-diagnostic period (from diagnosis to treatment initiation). Study length was variable ( $\leq 18$ months). The primary endpoint was time-adjusted semi-annual forced vital capacity (FVC) change measured during the peridiagnostic period using daily home spirometry in patients with idiopathic pulmonary fibrosis

\section{S. Jouneau}

Department of Respiratory Diseases, Competence Centre for Rare Pulmonary Diseases, Rennes University Hospital, 2 Rue Henri Le Guilloux, 35033 Rennes Cedex 9, France

S. Jouneau

Inserm, Irset (Institut de Recherche en Santé, Environnement et Travail)-UMR_S 1085, Univ Rennes, CHU Rennes, Avenue du Professeur Léon Bernard, 35000 Rennes, France

\section{A. G. Fois}

Department of Medical, Surgical and Experimental Sciences, University of Sassari, Piazza Università 21, 07100 Sassari, Sardinia, Italy

\section{O. Moran-Mendoza}

Department of Medicine, Queen's University, 99 University Avenue, Kingston, ON K7L 3N6, Canada 
(IPF). Secondary outcomes included changes in FVC (home spirometry) in patients with non-IPF ILD, changes in FVC (site spirometry), changes in physical functional capacity measured by daily home accelerometry and site 6-min walk distance (6MWD), and changes in patient-reported outcomes (PROs) in IPF or non-IPF ILD.

Results: Of the 178 patients enrolled in the study, 68 patients were diagnosed with IPF, 62 patients were diagnosed with non-IPF ILD, 9 patients received a non-ILD diagnosis and 39 patients did not receive a diagnosis. Technical and analytical issues led to problems in applying the prespecified linear regression model to analyse the home FVC data. Timeadjusted median (quartile $[\mathrm{Q}] 1, \mathrm{Q} 3$ ) semi-annual FVC change during the peri-diagnostic period measured using home and site spirometry, respectively, was $-147.7(-723.8,376.2) \mathrm{ml}$ and $-149.0(-314.6,163.9) \mathrm{ml}$ for IPF and 19.1 $(-194.9,519.0) \mathrm{ml}$ and $-23.4(-117.9,133.5)$ $\mathrm{ml}$ in non-IPF ILD. A greater decline in steps per day was observed for IPF versus non-IPF ILD, whereas an increase in 6MWD was observed for patients with IPF versus a decline in 6MWD for patients with non-IPF ILD. No clear patterns of disease behaviour were observed for IPF versus non-IPF ILD for PROs.

Conclusions: Despite home spirometry being feasible for most patients and centres, technical and analytical challenges in the home-based assessments prevented firm conclusions regarding disease behaviour. This highlights that further optimisation of the technology and analysis methods is required before widespread implementation.

\section{Trial Registration: NCT03261037.}

\section{S. Anees}

Schulich School of Medicine, University of Windsor, 401 Sunset Avenue, Windsor, ON N9B 3P4, Canada

M. Mirt · M. Bengus · F. Gilberg · K.-

U. Kirchgaessler

F. Hoffmann-La Roche, Ltd., Grenzacherstrasse 124, 4058 Basel, Switzerland

\section{Vancheri}

Department of Clinical and Experimental Medicine, Regional Referral Centre for Rare Lung Diseases, University Hospital Policlinico "G. Rodolico-San Marco", University of Catania, Via Santa Sofia 78, 95123 Catania, Italy
Keywords: Disease behaviour; Home spirometry; Idiopathic pulmonary fibrosis; Interstitial lung disease; Peri-diagnostic period

\section{Key Summary Points}

Why carry out this study?

Achieving a timely diagnosis of a specific ILD is important to inform prognosis and guide treatment decisions, as delays in treatment may negatively impact outcomes for patients.

Greater insight into disease behaviour may help to guide diagnosis and treatment decisions in patients with ILDs; however, there is currently a lack of evidence characterising ILD behaviour and progression during the early stages of disease.

The STARLINER study (NCT03261037) aimed to characterise disease behaviour in patients with suspected ILD during the peri-diagnostic period using daily home spirometry and accelerometry.

\section{What was learned from the study?}

During the peri-diagnostic period, differences in changes in FVC measured by home and site spirometry were observed between patients with IPF and patients with non-IPF ILD. A greater decline in steps per day was observed for IPF versus non-IPF ILD, whereas an increase in 6MWD was observed for patients with IPF versus a decline in 6MWD for patients with non-IPF ILD. No clear patterns of disease behaviour were observed for IPF versus non-IPF ILD for PROs.

Home spirometry was feasible for most patients with ILD; however, technical and analytical challenges in the home-based assessments prevented firm conclusions regarding disease behaviour and highlight the need for further optimisation of the technology and analysis methods before widespread implementation. 


\section{DIGITAL FEATURES}

This article is published with digital features, including two videos, to facilitate understanding of the article. To view digital features for this article go to https://doi.org/10.6084/m9. figshare.14578833.

\section{INTRODUCTION}

Interstitial lung diseases (ILDs) are a heterogeneous group of respiratory diseases characterised by fibrosis and/or inflammation of the lung [1]. The aetiology of ILDs is diverse. The cause of some ILDs is known, e.g., hypersensitivity pneumonitis resulting from environmental exposures or ILDs secondary to connective tissue diseases (CTD) [1]. However, in other cases, the causes are unknown, e.g., idiopathic pulmonary fibrosis (IPF) and other idiopathic interstitial pneumonias $[1,2]$.

Timely diagnosis of a specific ILD is important to inform prognosis and guide treatment plans $[1,3]$. The introduction of two antifibrotic treatments for IPF, pirfenidone and nintedanib, has increased the importance of early and accurate diagnosis for patients with fibrotic lung disease. Pirfenidone and nintedanib have been shown to modify the course of IPF by slowing IPF progression versus placebo, but cannot reverse existing fibrotic damage [4-6]. Therefore, early diagnosis and initiation of treatment are integral to slowing the irreversible decline in lung function experienced by patients with IPF. In other ILDs, early treatment of the underlying disease may also reverse disease, prevent or slow down disease progression, and relieve symptoms [7-9].

However, the diagnosis of an ILD is a complex process and many patients wait over 12 months for a diagnosis [10-12]. Reasons for delays are multifactorial and may include the presence of non-specific symptoms such as cough, dyspnoea and fatigue; a lack of evidence characterising ILD behaviour and progression during the early disease stages; and limited awareness about the prognosis and severity of ILDs among clinicians [13]. Many patients report one or more misdiagnoses prior to a final diagnosis [11] or geographical barriers to accessing specialised centres and multidisciplinary teams (MDTs) [14, 15].

It has been suggested that disease behaviour can be used to inform diagnosis, particularly in cases where reaching a diagnosis is difficult [16]; however, data on ILDs collected from around the time of diagnosis are scarce. Greater knowledge about ILD disease behaviour is needed to facilitate early and accurate diagnoses and guide treatment decisions. To develop a greater knowledge of ILD disease behaviour, it is important for frequent and detailed assessment of clinical measurements to be obtained as early as possible, particularly during the pre-diagnostic period. In clinical practice, the data available regarding disease behaviour may be limited during the pre-diagnostic period, and as clinic visits tend to occur in 3- or 6-month intervals, a more pragmatic approach may be required. Technologies such as home spirometry and accelerometry can allow these frequent measurements to be obtained as early as the prediagnostic period and may improve access to and quality of care for patients with ILD.

The STARLINER study aimed to assess disease behaviour in patients with suspected ILD during the peri-diagnostic period. The study used realtime home-based assessments, providing insights into the feasibility and utility of these technologies in patients with ILD.

\section{METHODS}

\section{Study Design and Participants}

STARLINER was an international, multicentre study conducted at community and tertiary clinical centres in Canada, France, Ireland, Italy, The Netherlands and Russia.

Eligible patients were aged $\geq 50$ years with suspected ILD, defined as radiological evidence of ILD in the presence of unexplained dyspnoea on exertion and/or cough. Patients were excluded if they had a history of clinically significant heart disease that could explain their symptoms, or a known history of CTD. 
Patients were followed throughout the peridiagnostic period, which comprised a pre-diagnostic period (from enrolment to diagnosis) and a post-diagnostic period (from diagnosis to treatment initiation) [17]. Patients left the study if they did not receive a diagnosis within 12 months of enrolment or if they received a non-ILD diagnosis. Patients who received a diagnosis of IPF or non-IPF ILD remained in the study until initiation of treatment for ILD or for a maximum of 6 months if treatment was not initiated. Therefore, the total duration of observation varied for each patient ( $\leq 18$ months). Please see Fig. 1 in the previously published study design manuscript (https://link.springer.com/article/10.1007/ s12325-018-0845-3\#Fig1) for further details.

Patients were managed at the discretion of their treating physician. Concomitant therapies for comorbidities were continued according to regular local practice and were documented in the electronic case report form (eCRF).

The methods of this study have been previously described [17].

\section{Compliance with Ethics Guidelines}

All patients gave written informed consent. The study conformed to the International Conference on Harmonisation (ICH) E6 and E2A guidelines for Good Clinical Practice and Clinical Safety Data Management, the principles of the Declaration of Helsinki or the laws and regulations of the country where the research was conducted, whichever afforded the greater protection to the individual. The study was approved by the master ethics committee (Stichting BEBO, Postbus 1004, 9400 BA, Assen, The Netherlands) and by ethics committees at all other participating institutes. This trial is registered with ClinicalTrials.gov (NCT03261037).

\section{Study Visits and Procedures}

Disease behaviour was assessed using a multidimensional approach that included pulmonary function, physical functional capacity, symptoms and quality of life (QoL) collected by patients at home and during site visits.
Patients performed one home measurement of forced vital capacity (FVC) each day using a portable handheld turbine spirometer (Spirobank $^{\circledR}$ Smart; Medical International Research, Rome, Italy). A second measurement was permitted if the technique of the first blow was not good, as detected by the spirometer and signalled to the patient. Patients' steps per day were continuously measured using an accelerometer resembling a watch (Steel HR; Withings, Issy-les-Moulineaux, France). There was no minimum duration that the patients were required to wear the accelerometer for during the study.

Site visits at baseline, diagnosis and end of study were the only mandatory site visits, and as the study was conducted under real-life conditions, the timing of these site visits was not pre-determined. Otherwise, patients were managed and seen according to the standard of care at the discretion of the treating clinician, which could lead to additional site visits. Site measurements included: FVC, 6-min walk test (6MWT) and patient-reported outcomes (PROs), including King's Brief Interstitial Lung Disease questionnaire (K-BILD), the EuroQoL 5-Dimensions 5-level index questionnaire (EQ-5D-5L), the Fatigue Assessment Scale (FAS), the modified Medical Research Council (mMRC) Dyspnoea Scale and three visual analogue scales (VAS) measuring cough, urge to cough and fatigue. Any further information collected as part of local standard of care was recorded in the eCRF. See Table S1 in the supplementary material for further details regarding mandatory outcome measures.

\section{Digital Collaboration Platform}

This study was facilitated by a novel digital collaboration platform, which has been described previously. Please see Fig. 2 in the previously published study design manuscript (https://link. springer.com/article/10.1007\%2Fs12325-0180845-3\#Fig2) for further details.

Each patient received a handheld spirometer, an accelerometer, a tablet computer and user manuals for each device at enrolment. Patients received training on how to use the devices and 
had access to a digital avatar to guide them through the use of the spirometers.

The tablet computers were linked to the digital collaboration platform to enable the treating physician to view their patient's realtime data, and patients also had the option to view their own real-time data. Additionally, the digital collaboration platform had an optional functionality to allow investigators from community centres to share clinical data and highresolution computed tomography (HRCT) images to obtain a second opinion from investigators from specialist tertiary ILD centres through virtual MDTs. Discussions between community and tertiary centres took place at any time during the study, and all diagnoses were at the discretion of the investigator.

The study sponsor did not have access to the digital collaboration platform and patient data collected by the home devices were anonymised before being transferred to the study database.

\section{Outcomes}

Information regarding diagnoses made during the study was collected, including, but not limited to, the percentage of patients diagnosed using a local MDT or via the virtual MDT function of the digital collaboration platform.

The primary endpoint was time-adjusted semi-annual change in FVC (ml) during the peri-diagnostic period measured using daily home spirometry in patients with IPF. The outcome was also analysed in patients with non-IPF ILD as a secondary endpoint.

Other secondary endpoints evaluated in patients with IPF and non-IPF ILD over the peridiagnostic period were: time-adjusted semi-annual change in FVC (ml) measured using site spirometry, the correlation between home and site FVC at baseline and time-adjusted semiannual changes in physical functional capacity measured by daily home accelerometry and site 6MWT. Changes in PRO scores over the peridiagnostic period were assessed, including EQ-5D-5L health state VAS and index scores, K-BILD total score and subscores, FAS, fatigue VAS, cough VAS, urge-to-cough VAS and mMRC
Dyspnoea Scale scores. Compliance with daily home measurements was also assessed.

There was no investigational medicinal product, and therefore there were no safety objectives; however, the incidences of respiratoryrelated and all-cause hospitalisations, respiratory-related and all-cause mortality, and acute exacerbations were reported.

\section{Statistical Analyses}

The planned sample size of 180 patients assumed that approximately $40 \%$ of patients who enrolled in the study would be diagnosed with IPF, 50\% would be diagnosed with non-IPF ILD and $10 \%$ would be diagnosed with a nonILD condition or would not have received a diagnosis within 12 months of enrolment.

The time-adjusted semi-annual FVC change measured using home spirometry was estimated for individual patients with at least three home spirometry measurements by applying a linear regression model. The estimated FVC change for each patient was then used to calculate the group mean and median FVC change.

An algorithm within the spirometers classified blows as acceptable or unacceptable manoeuvres (see Table S2 in the supplementary material). The primary analysis included only 'good blows' and an additional analysis included all 'acceptable blows'.

For secondary outcomes measuring FVC, 6-min walk distance (6MWD) and steps per day, the same methodology as for the primary endpoint was used to estimate time-adjusted semiannual changes during the peri-diagnostic period.

Sensitivity analyses excluding patients with less than 30 days of observation were performed for the primary and secondary outcomes detailed above.

For site measurements, baseline was defined as the measurement collected at enrolment.

To account for a learning curve for using the home devices, the first 7 days corresponding to the learning curve were removed; thus, for home spirometry and home accelerometry measurements, baseline was defined as the 
average of the daily home measurements collected during the next 7 consecutive days.

The correlation between baseline FVC measured using home and site spirometry was analysed by calculating Pearson's correlation taking the individual values for each patient into account.

Changes in PROs and the incidences of respiratory-related hospitalisations, all-cause hospitalisations, acute exacerbations, respiratoryrelated mortality and all-cause mortality, and the percentage of patients diagnosed using an MDT were summarised descriptively.

The percentage per patient of days with at least one home spirometry measurement was calculated as the ratio of the number of days with at least one measurement and the number of days the patient was observed within the study. The percentage of patients with at least one gap in daily home spirometry or accelerometry measurements (a gap in daily home measurements was defined as $\geq 7$ consecutive days of missing measurements) and the duration of gaps were summarised descriptively.

\section{RESULTS}

\section{Study Participants}

Between 25 December 2017 and 4 February 2019, 178 patients were enrolled from 37 centres ( 24 community and 13 tertiary centres). In total, $68(38.2 \%)$ patients were diagnosed with IPF, $62(34.8 \%)$ patients were diagnosed with non-IPF ILD, 9 (5.1\%) patients were diagnosed with a non-ILD condition, and 39 (21.9\%) patients did not receive a diagnosis because they had either not received a diagnosis within 12 months of enrolment $(11 / 39$ [28.2\%]) or because they discontinued the study early before being diagnosed (28/39 [71.8\%]; Fig. 1).

Baseline characteristics were generally similar across the diagnostic subgroups; however, a higher percentage of patients with IPF were male and had a previous history of smoking compared with patients with non-IPF ILD (Table 1).

The virtual MDT function of the digital collaboration platform was used for diagnosis in $14.7 \%$ and $17.7 \%$ of patients diagnosed with IPF

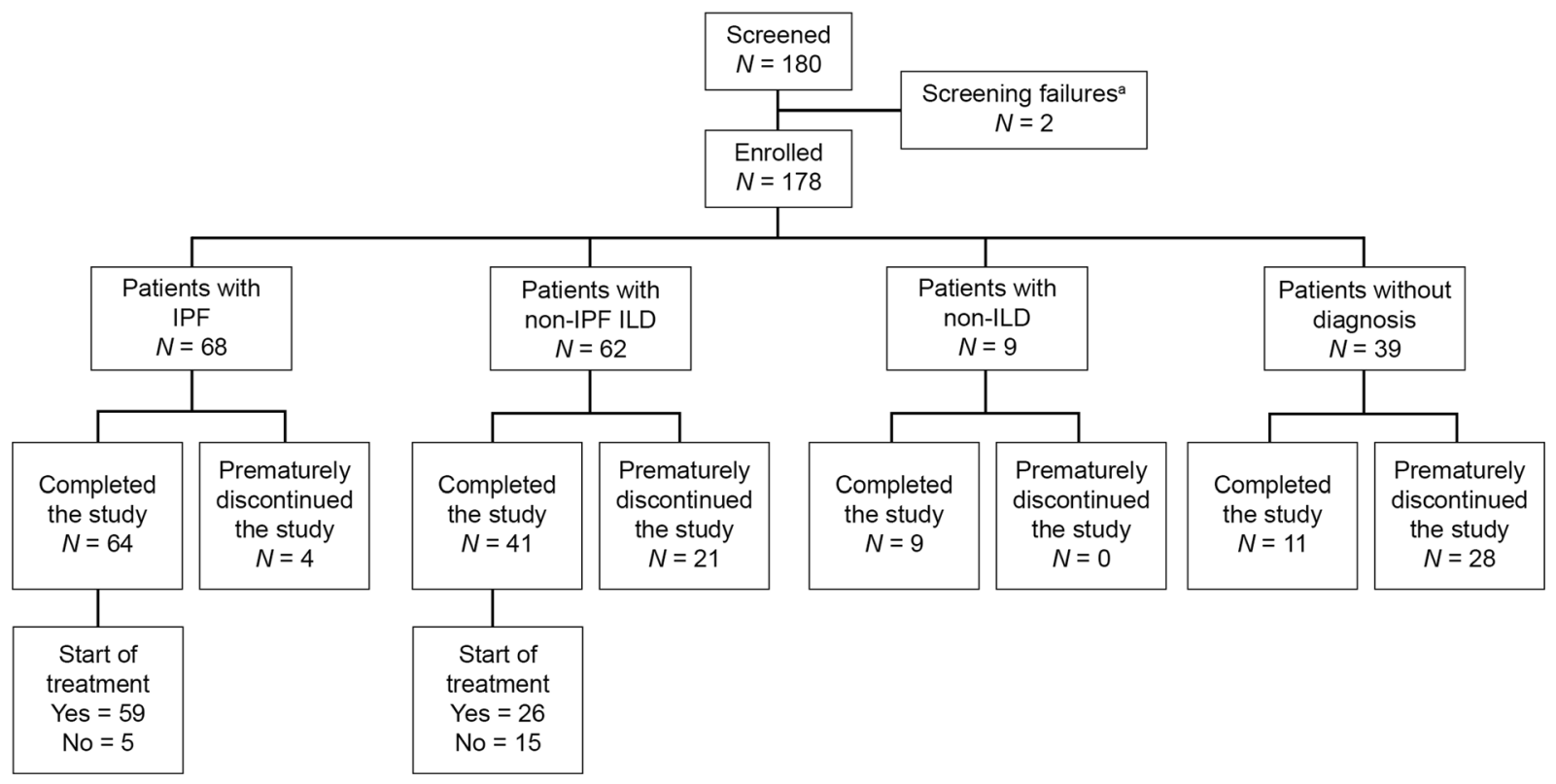

Fig. 1 Patient disposition. ILD interstitial lung disease, $I P F$ idiopathic pulmonary fibrosis. ${ }^{a}$ One patient failed screening because the investigator did not consider them able to comply with the study protocol, e.g., the ability to

use the provided spirometer and tablet, and the ability to fill in the required patient-reported outcomes questionnaires. The other screening failure was due to confirmation of a large B-cell malignancy 
Table 1 Baseline demographics and clinical characteristics

\begin{tabular}{|c|c|c|}
\hline & $\begin{array}{l}\text { IPF } \\
(N=68)\end{array}$ & $\begin{array}{l}\text { Non-IPF ILD } \\
(N=62)\end{array}$ \\
\hline $\begin{array}{l}\text { Age at enrolment, } \\
\text { years }\end{array}$ & $\begin{array}{l}69.5(65.0 \\
75.0)\end{array}$ & $\begin{array}{c}65.5(56.0 \\
74.0)\end{array}$ \\
\hline Male, $n(\%)$ & $49(72.1)$ & $30(48.4)$ \\
\hline \multicolumn{3}{|l|}{ Race, $n(\%)$} \\
\hline White & $62(91.2)$ & $50(80.6)$ \\
\hline $\begin{array}{l}\text { American Indian or } \\
\text { Alaskan Native }\end{array}$ & $1(1.5)$ & 0 \\
\hline Missing/unknown & $5(7.4)$ & $12(19.4)$ \\
\hline \multicolumn{3}{|l|}{ Smoking history, $n$ (\%) } \\
\hline Never & $19(27.9)$ & $28(45.2)$ \\
\hline Current & $4(5.9)$ & $6(9.7)$ \\
\hline Previous & $45(66.2)$ & $28(45.2)$ \\
\hline \multicolumn{3}{|l|}{ Type of site, $n(\%)$} \\
\hline Community & $49(72.1)$ & $45(72.6)$ \\
\hline Tertiary & $19(27.9)$ & $17(27.4)$ \\
\hline \multicolumn{3}{|l|}{ FVC, 1} \\
\hline Home $^{a}$ & $2.8(2.1,3.4)^{b}$ & $2.6(1.8,3.3)^{c}$ \\
\hline Site & $2.8(2.3,3.4)^{\mathrm{d}}$ & $2.8(1.9,3.4)^{\mathrm{e}}$ \\
\hline \multicolumn{3}{|c|}{ Physical functional capacity } \\
\hline Steps per day ${ }^{\mathrm{a}}$ & $\begin{array}{l}3178.4 \\
\quad(1493.7 \\
5645.4)^{d}\end{array}$ & $\begin{array}{l}3778.4 \\
\quad(2182.9 \\
6034.0)^{\mathrm{e}}\end{array}$ \\
\hline 6MWD, m & $\begin{array}{l}449.0(370.0 \\
510.0)^{\mathrm{d}}\end{array}$ & $\begin{array}{l}448.5(316.5 \\
531.0)^{c}\end{array}$ \\
\hline \multicolumn{3}{|l|}{ EQ-5D-5L, mean (SD) } \\
\hline Health state VAS ${ }^{f}$ & $71.7(16.0)$ & $67.4(20.1)^{\mathrm{e}}$ \\
\hline EQ-5D index & $0.8(0.1)$ & $0.79(0.1)$ \\
\hline \multicolumn{3}{|l|}{ K-BILD, mean (SD) } \\
\hline Total score & $59.7(12.9)$ & $56.3(10.3)$ \\
\hline Psychological & $60.6(17.6)$ & $55.7(14.2)$ \\
\hline $\begin{array}{l}\text { Breathlessness and } \\
\text { activities }\end{array}$ & $50.1(20.8)$ & $45.3(19.0)$ \\
\hline Chest symptoms & $70.0(21.6)$ & $69.5(20.1)$ \\
\hline
\end{tabular}

Table 1 continued

\begin{tabular}{|c|c|c|}
\hline & $\begin{array}{l}\text { IPF } \\
(N=68)\end{array}$ & $\begin{array}{l}\text { Non-IPF ILD } \\
(N=62)\end{array}$ \\
\hline FAS, mean (SD) & $21.8(6.4)^{\mathrm{g}}$ & $21.8(6.9)^{c}$ \\
\hline $\begin{array}{l}\text { Fatigue } \mathrm{VAS}^{\mathrm{f}} \text {, mean } \\
\quad(\mathrm{SD})\end{array}$ & $38.4(27.2)$ & $39.4(24.3)^{c}$ \\
\hline $\begin{array}{l}\text { Cough-VAS }{ }^{f} \text {, mean } \\
\text { (SD) }\end{array}$ & $34.0(25.4)$ & $26.9(20.7)^{c}$ \\
\hline $\begin{array}{l}\text { Urge-to-cough VAS } \\
\text { mean }(\mathrm{SD})\end{array}$ & $33.3(25.4)$ & $28.6(21.5)^{c}$ \\
\hline
\end{tabular}

Data are median (Q1, Q3) unless otherwise specified 6MWD 6-min walk distance, EQ-5D EuroQoL 5 Dimensions questionnaire, EQ-5D-5L EuroQoL 5 Dimensions 5-level index questionnaire, $F A S$ Fatigue Assessment Scale, FVC forced vital capacity, ILD interstitial lung disease, $I P F$ idiopathic pulmonary fibrosis, $K-B I L D$ King's Brief Interstitial Lung Disease questionnaire, $Q$ quartile, $S D$ standard deviation, $V A S$ visual analogue scale

a To account for a learning curve for using the at-home devices, the first 7 days corresponding to the learning curve were removed; thus, for home spirometry and home accelerometry measurements, baseline was defined as the average of the daily home measurements collected during the next 7 consecutive days

b $n=65$

c $n=60$

d $n=66$

e $n=61$

${ }^{f}$ Measured on a scale from 0 to 100

g $n=67$

and non-IPF ILD, respectively. A local MDT was used in $70.6 \%$ of patients with IPF and $59.7 \%$ of patients with non-IPF ILD, whereas an MDT was not used in $14.7 \%$ and $22.6 \%$ of patients with IPF and non-IPF ILD, respectively. See Table S3 and Table $S 4$ in the supplementary material for further details on the non-IPF ILD diagnoses and the assessments used to make a diagnosis of IPF or non-IPF ILD.

The overall median (minimum-maximum) time from enrolment to diagnosis was 10.9 (0.6-53.3) weeks in patients with IPF and 20.7 


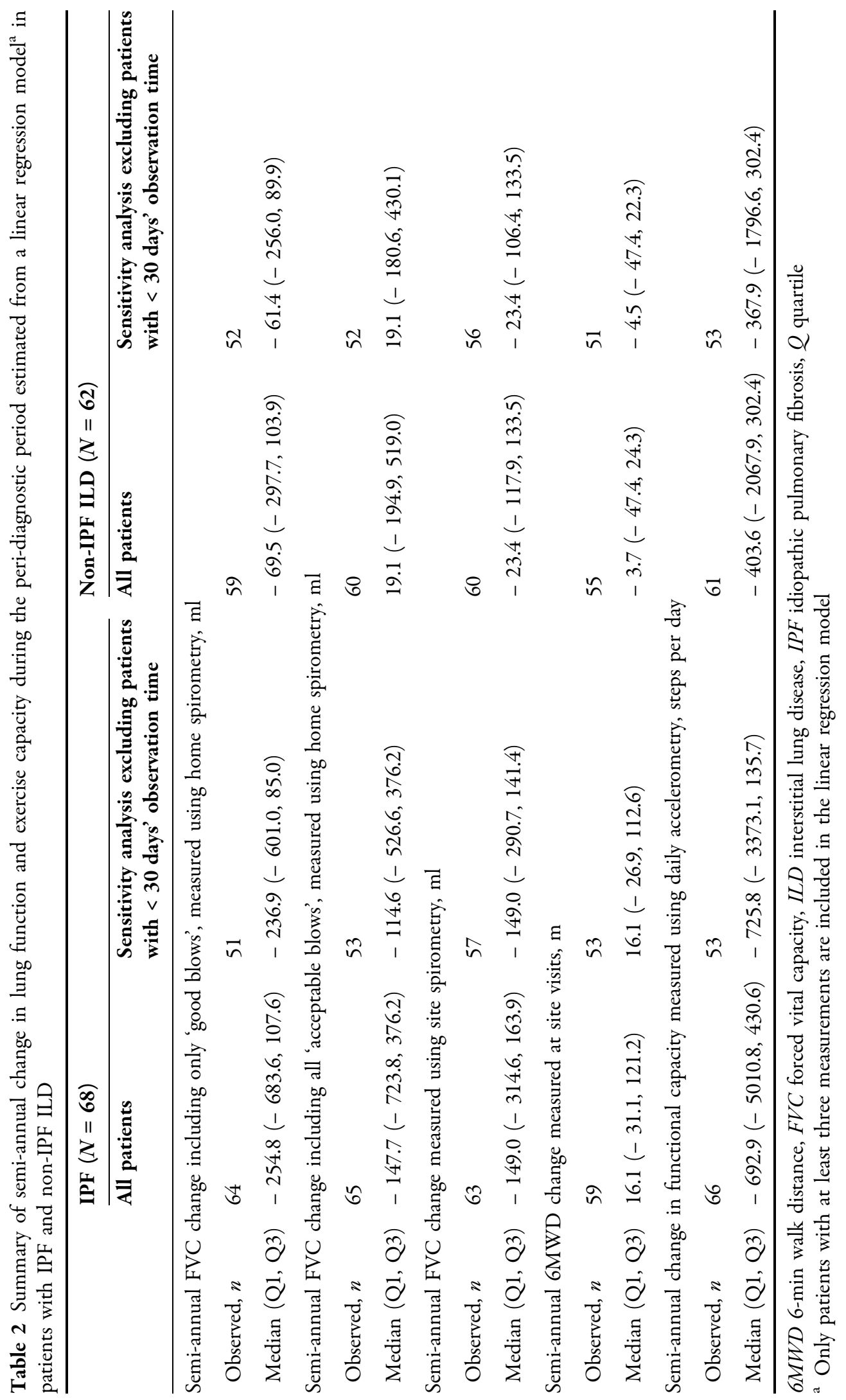



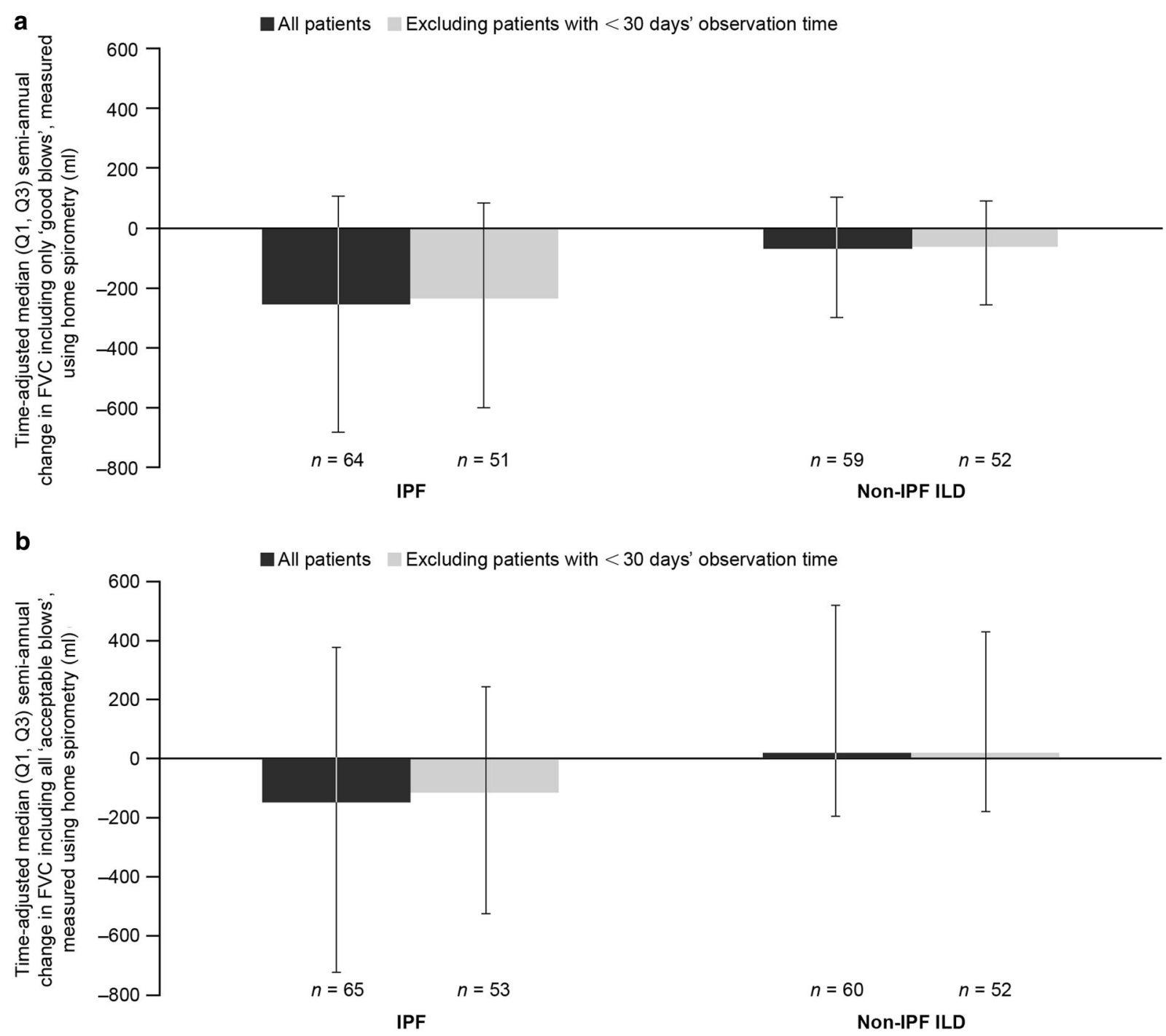

Fig. 2 Time-adjusted semi-annual FVC change during the peri-diagnostic period in patients with IPF and non-IPF ILD, measured by home spirometry including (a) only

(0.9-52.9) weeks in patients with non-IPF ILD. For patients enrolled at community sites, the median (minimum-maximum) time from enrolment to diagnosis was 10.7 (0.6-53.3) weeks in patients with IPF and 21.3 (0.9-51.4) weeks in patients with non-IPF ILD. For patients enrolled at tertiary sites, the median (minimum-maximum) time from enrolment to diagnosis was 11.0 (3.1-38.9) weeks in patients with IPF and $19.6(1.0-52.9)$ weeks in patients with non-IPF ILD. 'good blows' and (b) all 'acceptable blows'. $F V C$ forced vital capacity, ILD interstitial lung disease, IPF idiopathic pulmonary fibrosis, $Q$ quartile

In total, $59(86.8 \%)$ patients with IPF and $26(41.9 \%)$ patients with non-IPF ILD were initiated on treatment within 6 months of diagnosis. The overall median (minimummaximum) time from diagnosis to treatment initiation was $1.4(0.1-27.3)$ weeks in patients with IPF and $6.6(0.1-27.9)$ weeks in patients with non-IPF ILD. For patients enrolled at community sites, the median (minimummaximum) time from diagnosis to treatment initiation was $2.0(0.1-26.4)$ weeks in patients with IPF and $0.3(0.1-27.9)$ weeks in patients 
Table 3 Changes from baseline in PROs during the peri-diagnostic period in patients with IPF and non-IPF ILD

\begin{tabular}{lll}
\hline & IPF $(\boldsymbol{N}=\mathbf{6 8})$ & Non-IPF ILD $(\boldsymbol{N}=\mathbf{6 2})$ \\
\hline EQ-5D-5L & $-2.0(15.4)^{\mathrm{b}}$ & $3.1(15.3)^{\mathrm{c}}$ \\
Health state VAS & $-0.02(0.14)^{\mathrm{b}}$ & $0.01(0.12)^{\mathrm{d}}$ \\
EQ-5D index & & \\
K-BILD & $-0.45(9.71)^{\mathrm{b}}$ & $3.98(11.85)^{\mathrm{d}}$ \\
Total score & $-1.95(14.07)^{\mathrm{b}}$ & $6.79(17.35)^{\mathrm{d}}$ \\
Psychological & $-1.24(17.74)^{\mathrm{b}}$ & $2.86(17.08)^{\mathrm{d}}$ \\
Breathlessness and activities & $1.30(20.81)^{\mathrm{b}}$ & $-0.24(20.23)^{\mathrm{d}}$ \\
Chest symptoms & $0.1(5.0)^{\mathrm{e}}$ & $-0.2(5.5)^{\mathrm{f}}$ \\
FAS & $-1.0(27.5)^{\mathrm{b}}$ & $-5.8(23.5)^{\mathrm{g}}$ \\
Fatigue VAS & $0.1(28.8)^{\mathrm{b}}$ & $-3.8(21.2)^{\mathrm{g}}$ \\
Cough VAS & $-0.9(28.6)^{\mathrm{b}}$ & $-5.2(22.0)^{\mathrm{g}}$ \\
Urge-to-cough VAS $^{\mathrm{a}}$ & &
\end{tabular}

Data are mean (SD) unless otherwise specified. For EQ-5D and K-BILD scores, a higher score indicates better QoL. For FAS, a higher score indicates more fatigue. For fatigue VAS, cough VAS and urge-to-cough VAS, a higher score indicates worse fatigue, worse cough and greater urge to cough, respectively. As the duration of observation for each patient was variable, in cases where more than one visit was performed during the pre-/post-diagnostic periods, mean values of these visits were used for derivation of descriptive statistics

EQ-5D EuroQoL 5 Dimensions questionnaire, EQ-5D-5L EuroQoL 5 Dimensions 5-level index questionnaire, FAS Fatigue Assessment Scale, $I L D$ interstitial lung disease, IPF idiopathic pulmonary fibrosis, $K$-BILD King's Brief Interstitial Lung Disease questionnaire, $P R O$ patient-reported outcome, $Q D L$ quality of life, $S D$ standard deviation, VAS visual analogue scale

${ }^{\text {a }}$ Measured on a scale from 0 to 100

b $n=63$

c $n=59$

d $n=60$

e $n=62$

f $n=56$

g $n=58$

with non-IPF ILD. For patients enrolled at tertiary sites, the median (minimum-maximum) time from diagnosis to treatment initiation was $1.4(0.1-27.3)$ weeks in patients with IPF and non-calculable (0.1-27.1) weeks in patients with non-IPF ILD. Further details regarding patient disposition and treatment initiation are presented in Table S5 of the supplementary material.

\section{Primary Endpoint}

The analysis of the home spirometry data was affected by outliers, primarily due to patients with few data points or short observation periods, resulting in individual slope estimates that were not normally distributed. Therefore, presentation of mean group values, as prespecified, was not appropriate and median was chosen as a more suitable statistic for skewed data. For completeness, the prespecified mean data are 
provided in Table S6 of the supplementary material. Additionally, the prespecified primary analysis plan included only 'good blows'; however, as 'acceptable blows' were thought to be more representative of clinical practice, these data are discussed in the main text. The data including only 'good blows' can be found in Table 2 in the main manuscript and Table S6 of the supplementary material.

Time-adjusted median (quartile [Q]1, Q3) semi-annual change in FVC measured using home spirometry during the peri-diagnostic period in patients diagnosed with IPF was - 147.7 (- 723.8, 376.2) $\mathrm{ml}$ when including all 'acceptable blows' (Table 2; Fig. 2).

\section{Secondary Endpoints}

Time-adjusted median (Q1, Q3) semi-annual change in FVC measured using home spirometry during the peri-diagnostic period in patients with non-IPF ILD was 19.1 (-194.9, 519.0) ml when including all 'acceptable blows' (Table 2; Fig. 2).

Time-adjusted median (Q1, Q3) semi-annual change in FVC measured using site spirometry during the peri-diagnostic period was -149.0 $(-314.6,163.9) \mathrm{ml}$ in patients with IPF and $-23.4(-117.9,133.5) \mathrm{ml}$ in patients with nonIPF ILD (Table 2).

The correlation between baseline home and site FVC (including only 'good blows') was moderate/strong (Pearson's correlation coefficient $=0.71$ and 0.69 for patients with IPF and non-IPF ILD, respectively).

Time-adjusted median (Q1, Q3) semi-annual change in steps per day during the peri-diagnostic period was $-692.9(-5010.8,430.6)$ steps in patients with IPF and $-403.6(-2067.9$, 302.4) steps in patients with non-IPF ILD (Table 2).

Time-adjusted median (Q1, Q3) semi-annual change in 6MWD during the peri-diagnostic period was $16.1(-31.1,121.2) \mathrm{m}$ in patients with IPF and $-3.7(-47.4,24.3) \mathrm{m}$ in patients with non-IPF ILD (Table 2).

In sensitivity analyses excluding patients with $<30$ days of observation, time-adjusted semi-annual FVC change measured using home spirometry, including all 'acceptable blows', during the peri-diagnostic period was - 114.6 $(-526.6,376.2) \mathrm{ml}$ in patients with IPF and 19.1 $(-180.6,430.1) \mathrm{ml}$ in patients with non-IPF ILD. Further details regarding sensitivity analyses of lung function and exercise capacity outcomes in patients with IPF and non-IPF ILD excluding patients with $<30$ days of observation are presented in Table 2, Fig. 2 and Table S6 of the supplementary material.

During the peri-diagnostic period, mean (standard deviation [SD]) change from baseline in EQ-5D-5L health state VAS score was a deterioration of -2.0 (15.4) in patients with IPF and an improvement of 3.1 (15.3) in patients with non-IPF ILD (Table 3). Mean (SD) change from baseline in EQ-5D index score was a deterioration of $-0.02(0.14)$ for patients with IPF and an improvement of $0.01(0.12)$ in patients with non-IPF ILD (Table 3). Mean (SD) change from baseline in K-BILD total score was a deterioration of -0.45 (9.71) for patients with IPF and an improvement of 3.98 (11.85) in patients with non-IPF ILD (Table 3).

Results for K-BILD subscores, FAS, fatigue VAS, cough VAS and urge-to-cough VAS are also presented in Table 3. Results for mMRC Dyspnoea Scale scores are presented in Table S7 of the supplementary material.

The incidence rate of non-elective hospitalisations was low. In total, six (8.8\%) and two (2.9\%) patients diagnosed with IPF experienced at least one all-cause hospitalisation during the pre-diagnostic (11 events) and post-diagnostic (two events) period, respectively. The corresponding values for respiratory-related hospitalisations were four (5.9\%) patients (five events) and 0 patients. In total, three (4.8\%) and 0 patients diagnosed with non-IPF ILD experienced at least one all-cause hospitalisation during the pre-diagnostic (six events) and postdiagnostic period, respectively. Respective values for respiratory-related hospitalisations were three $(4.8 \%)$ patients (five events) and 0 patients. One patient with IPF experienced a single acute exacerbation during the pre-diagnostic period. No deaths were recorded during this study. 


\section{Quality of Home Assessments}

The total number of blows performed during the study per cohort was 8647 for patients with IPF and 12,167 for patients with non-IPF ILD. Of these, $54.0 \%$ and $60.2 \%$, respectively, were categorised as 'good blows'; $19.6 \%$ and 19.5\%, respectively, were categorised as 'acceptable blows except good blows'; and $26.4 \%$ and $20.2 \%$, respectively, were categorised as 'bad blows' by the spirometer algorithm. The median (Q1, Q3) duration of observation was 15.4 (8.5, 30.6) weeks in patients with IPF and 28.5 (13.3, 46.7) weeks in patients with non-IPF ILD.

\section{Compliance with Home Assessments}

Compliance with home spirometry, including all blows, is presented for IPF and non-IPF ILD in Fig. S1 of the supplementary material. During the peri-diagnostic period, the median (Q1, Q3) adherence to home spirometry, defined as the percentage per patient of days with $\geq 1$ home spirometry blow, was $84.8 \%(73.2,93.2)$ for IPF and $87.5 \%(72.6,95.2)$ for non-IPF ILD. During the peri-diagnostic period, $50.0 \%$ of patients with IPF and $53.2 \%$ of patients with non-IPF ILD had at least one gap in daily home spirometry measurements (a gap in daily home measurements was defined as $\geq 7$ consecutive days of missing measurements). The median (Q1, Q3) duration of gaps was $20.6(11.0,41.0)$ days in patients with IPF and 21.0 (16.0, 43.0) days in patients with non-IPF ILD. During the peri-diagnostic period, $60.3 \%$ of patients with IPF and $75.8 \%$ of patients with non-IPF ILD had at least one gap in daily home accelerometry measurements. The median (Q1, Q3) duration of gaps was $27.5(18.0,52.0)$ days in patients with IPF and $26.0(14.0,66.0)$ days in patients with nonIPF ILD. The mean (95\% confidence interval [CI]) percentage of days with at least one blow was $77.7 \%(72.0,83.3)$ for patients with IPF and $78.1 \%(72.1,84.1)$ for patients with non-IPF ILD.

\section{DISCUSSION}

The STARLINER study aimed to utilise a digital ecosystem to investigate disease behaviour during the peri-diagnostic period in patients with suspected ILD. This study is the first to collect prospective data on ILD disease behaviour during the time period before diagnosis and treatment initiation. Daily home spirometry and accelerometry allowed more frequent data collection and minimised the number of mandatory site visits required to measure lung function and exercise capacity. During the peridiagnostic period, differences in changes in FVC measured by home and site spirometry were observed between patients with IPF and patients with non-IPF ILD. A greater decline in steps per day was observed for IPF versus non-IPF ILD, whereas an increase in 6MWD was observed for patients with IPF versus a decline in 6MWD for patients with non-IPF ILD. No clear patterns of disease behaviour were observed for IPF versus non-IPF ILD for PROs. Technical and analytical difficulties in the home-based assessments prevented firm conclusions regarding disease behaviour in these diagnostic subgroups and highlight the need for further optimisation of the technology.

Similar to previous studies utilising home spirometry, a number of technical and analytical difficulties were encountered during this study [18]. Connectivity issues between the patients' devices and the digital platform were encountered, which either prevented patients from performing the home-based measurements or prevented the transfer of data from the devices to the digital platform. There was also no direct helpdesk support available to help patients overcome these issues, and clinicians had to contact the helpdesk on behalf of patients. Additionally, the algorithm within the home spirometers determined blow quality based on technique rather than the obtained value, thus allowing clinically implausible values to be accepted as 'good blows'. Furthermore, some patients only had a small number of observations collected over a short time period during the study, which led to problems in applying the prespecified linear regression model to analyse the home FVC data, leading to clinically implausible predicted changes in FVC for individual patients, with a subsequent effect on group means. Therefore, whilst home spirometry can be a useful tool, the technical 
and analytical issues experienced in this study highlight a clear need for further optimisation of the technology. It is important to determine the most accurate type of spirometer, improve the algorithms within spirometers to identify and discard implausible lung-function values and ensure proper calibration of home spirometers to prevent inaccurate measurements $[19,20]$, and further explore how to analyse home spirometry data in clinical trials [21]. The availability of direct helpdesk support for patients in their local language may help to reduce any technical issues and increase compliance with home measurements in future studies [22]. Furthermore, it is necessary to develop standardised criteria for home spirometry, including frequency of measurements, how many blows may be required per day and suitable acceptability and repeatability criteria for uncontrolled environmental conditions.

Despite the technical issues described above, we demonstrated that in line with previous studies, daily home spirometry was feasible for most patients; however, compliance was variable [22-26]. Home measurements can allow more frequent data collection and minimise the number of mandatory site visits required to measure lung function and exercise capacity. During the COVID-19 pandemic, several components of the diagnosis and management process in patients with ILD have been impacted as a result of minimising face-to-face interactions between healthcare workers and patients with ILD. Moreover, many clinical consultations have taken place via videoconferencing, and pulmonary function tests, which have a high risk of spreading infection, have been restricted [27]. Therefore, optimisation of smartphone technologies, home spirometry technologies and virtual platforms, like the one used in this study, is crucial to maintain or improve the level of care surrounding the diagnosis, monitoring and management of patients with ILD, particularly during the COVID-19 pandemic $[21,27,28]$.

A number of insights were gained from this study. First, when measured by home spirometry, patients with IPF experienced a decline in FVC during the peri-diagnostic period, whereas an increase in FVC was observed for patients with non-IPF ILD. In line with the home spirometry data, patients with IPF experienced a decline in FVC during the peri-diagnostic period, when measured by site spirometry. A decline in FVC was also observed for patients with non-IPF ILD when measured by site spirometry; however, patients with IPF experienced a greater decline in FVC versus patients with non-IPF ILD. A greater decline in steps per day was observed for IPF versus non-IPF ILD, whereas an increase in 6MWD was observed for patients with IPF versus a decline in 6MWD for patients with non-IPF ILD. Although the results cannot be directly compared, the observation that the deterioration in steps per day was not mirrored by the 6MWD results may reflect that the 6MWT is a measure of what patients can do in a clinical environment, whereas daily accelerometry is a measure of what patients do under normal circumstances at home. No clear patterns of disease behaviour were observed for IPF versus non-IPF ILD for PROs. In patients with IPF, EQ-5D-5L health state VAS score and K-BILD total score tended to worsen over time, but these changes did not exceed previously reported ranges of the minimal clinically important difference (MCID) [29, 30]. In patients with non-IPF ILD, the improvements from baseline in EQ-5D-5L health state VAS score and K-BILD total score were not greater than the previously reported range of MCIDs $[29,30]$. However, in patients with IPF, the deterioration from baseline in EQ-5D index score was within the reported MCID range of 0.005-0.054 [29] and the same applied for the improvement from baseline in EQ-5D index score in patients with non-IPF ILD [29]. Therefore, these data suggest that patient symptoms and well-being, measured by structured PROs, may also provide insights into disease behaviour during the diagnostic trajectory, mimicking clinical practice where a combination of these factors is considered when forming a diagnosis.

Whilst technical and analytical issues prevent firm conclusions about differences in disease behaviour between IPF and non-IPF ILD in this study, further investigations may be able to clarify differences in disease behaviour between these diagnostic subgroups during the peri- 
diagnostic period and their additive value in classifying ILDs and forming diagnoses in the future [31]. Although disease behaviour alone would not form the basis of a diagnosis, it could, alongside other clinical and radiological information, help clinicians to form working diagnoses and to re-evaluate them when longitudinal disease behaviour is discordant with the working diagnosis [16, 31].

A second key observation was that the median time from enrolment to diagnosis for nonIPF ILD was twice that reported for IPF (20.7 weeks vs. 10.9 weeks, respectively). This finding may reflect that the diagnosis of IPF can be relatively straightforward when patients present with classical symptoms, HRCT images and no relevant exposures. In comparison, the radiological picture can be less clear in non-IPF ILDs, and there may be a need for further diagnostic assessments, e.g., bronchoalveolar lavage or biopsy, or involvement of other specialists, e.g., rheumatologists in patients with suspected CTD. In clinical practice, diagnostic delays and misdiagnosis of ILDs have been well documented [10-12] and can have many negative effects on patients, including the need for consultations with multiple clinicians, repetition of diagnostic tests and poorer long-term outcomes $[11,12]$. Importantly, delays in diagnosis can prevent the initiation of treatments, deny patients the chance to take part in clinical trials and cause anxiety for patients and their families.

A number of limitations should be considered when interpreting the results of this study. It should be noted that it was not possible to analyse any data for calorie expenditure during this study as technical difficulties with the devices used for functional capacity measurements led to unreliable data being collected. Additionally, some problems were experienced when trying to upload or view HRCT images on the digital collaboration platform. These problems may have prevented full use of the virtual MDT function and potentially impacted the number of patients who were diagnosed. The percentage of patients diagnosed with a nonILD, or who received no diagnosis, exceeded the $10 \%$ assumed in the sample-size calculation. Particularly, the number of patients with no diagnosis $(N=39)$ was higher than expected, which may have impacted the median time to diagnosis; however, only 11 patients were excluded from the study because they had not received a diagnosis after 12 months in the study-the remaining 28 patients with no diagnosis discontinued the study prematurely and may have received a diagnosis had they remained in the study. The presence of a subgroup of patients without diagnosis after 12 months highlights the unmet need for an earlier and accurate diagnosis in these patients. Furthermore, it was not possible to perform correlation analyses between home and site FVC in patients with IPF and non-IPF ILD over time, as the only mandatory site visits were at baseline, diagnosis and end of study, and these site visits did not have a prespecified schedule. Additionally, there may have been a selection bias for patients who were more interested in, or more familiar with, online technologies, as only 1 of the 180 eligible patients failed screening because of not being able to use the provided equipment and complete the PRO questionnaires. It is, however, unlikely that this influenced the diagnostic trajectory of the patients included in the study. Similarly, only community sites interested in home monitoring in ILD would have participated in this study, which may have led to a selection bias; however, this bias is also present in many other clinical trials, and the inclusion criteria in this study were broad, which may have resulted in a patient population and diagnostic practices that are more representative of the ILD field. Finally, the widespread availability of the technology used in this study may be limited by financial barriers in some healthcare systems; however, the COVID-19 pandemic is forcing policy makers to consider new and sustainable care models.

In conclusion, this is the first study to investigate disease behaviour in patients with suspected ILD during the peri-diagnostic period using real-time, home-based assessments. During this study, differences in FVC change measured by home and site spirometry were observed between patients with IPF and patients with non-IPF ILD. A greater decline in steps per day was observed for IPF versus non-IPF ILD, whereas an increase in 6MWD was observed for 
patients with IPF versus a decline in 6MWD for patients with non-IPF ILD. It is not clear why the results for steps per day and 6MWD were discordant; however, it may reflect that these measurements capture different aspects of physical activity. No clear patterns of disease behaviour were observed for IPF versus non-IPF ILD for PROs. Technical and analytical difficulties in the home-based assessments prevented firm conclusions regarding disease behaviour in these diagnostic subgroups and highlight the need for further optimisation of the technology. Nevertheless, use of the technology in this study was feasible for most patients and centres. Further optimisation of the home-based assessments and virtual MDT platforms for clinicians and patients, such as the one used in this study, is required before these can be used in clinical practice to facilitate collaboration among patients, community centres and tertiary centres to improve the timely access to diagnosis and treatment for patients.

\section{ACKNOWLEDGEMENTS}

We would like to thank the patients, their family members and participating staff at all of the study centres. We would also like to thank Sonja Rohner of F. Hoffmann-La Roche, Ltd., for her ongoing support with this study.

Funding. This study is sponsored by F. Hoffmann-La Roche, Ltd. The Advances in Therapy Rapid Service Fees and Open Access Fees are funded by F. Hoffmann-La Roche, Ltd.

Authorship. All named authors meet the International Committee of Medical Journal Editors (ICMJE) criteria for authorship of this article, take responsibility for the integrity of the work as a whole and have given their approval for this final version to be published.

Author Contributions. MSW, EB, CV, MTH, CCM, SJ, AGF, OM-M, SA, MM, FG, MB, K-UK and $\mathrm{CV}$ were involved in the conception and/or design of the work, the interpretation of study results, and contributed to the manuscript from the outset. Data analysis was performed by FG. All authors read and approved the final draft, and vouch for the accuracy of the content included in the final manuscript.

Medical Writing, Editorial and Other Assistance. Medical writing support was provided by Catherine Stanton and Leigh Clements, PhD, of CMC AFFINITY, McCann Health Medical Communications, funded by F. Hoffmann-La Roche, Ltd.

Prior Presentation. Some of the results of the study were presented as a poster at the virtual European Respiratory Society (ERS) Congress (7 September to 9 September 2020).

Disclosures. Marlies S Wijsenbeek has received unrestricted grants and speaker fees from Boehringer Ingelheim and F. Hoffmann-La Roche, Ltd., and advisory/DSM board fees from BMS, Galapagos, Horizon, Novartis, Respivant and Safara. All grants and fees were paid to the Erasmus University Medical Center in Rotterdam, The Netherlands. Elisabeth Bendstrup has received unrestricted grants, speaker fees and advisory board fees from Boehringer Ingelheim and F. Hoffmann-La Roche, Ltd. Claudia Valenzuela has received speaker fees and advisory board fees from Boehringer Ingelheim, F. Hoffmann-La Roche, Ltd. and Galapagos. Michael T Henry has received speaker fees and/ or advisory board fees from Boehringer Ingelheim, F. Hoffmann-La Roche, Ltd., GlaxoSmithKline and Menarini. Catharina C Moor has received unrestricted grants and speaker and/or advisory board fees from Boehringer Ingelheim. All grants and fees were paid to the Erasmus University Medical Center in Rotterdam, The Netherlands. Stéphane Jouneau has received fees, funding or reimbursement for national and international conferences, boards, expert or opinion groups and research projects over the past 5 years from Actelion, AIRB, AstraZeneca, Bellerophon Therapeutics, Biogen, BMS, Boehringer Ingelheim, Chiesi, F. Hoffmann-La Roche, Ltd., FibroGen, Galecto Biotech, Genzyme, Gilead, GlaxoSmithKline, LVL, Mundipharma, Novartis, Pfizer, PharmOlam, Pliant Therapeutics, Sanofi and Savara- 
Serendex. Alessandro G Fois has received speaker and/or advisory board fees from Boehringer Ingelheim and F. Hoffmann-La Roche, Ltd. Onofre Moran-Mendoza reports research funding, honoraria and clinical trial participation fees from F. Hoffmann-La Roche, Ltd., and consultancy fees from Boehringer Ingelheim and F. Hoffmann-La Roche, Ltd. Syed Anees has received speaker fees and/or advisory board fees from Boehringer Ingelheim, F. Hoffmann-La Roche, Ltd., GlaxoSmithKline and Novartis. Mirela Mirt and Frank Gilberg are employees of F. Hoffmann-La Roche, Ltd. Monica Bengus and Klaus-Uwe Kirchgaessler are employees and shareholders of F. Hoffmann-La Roche, Ltd. Carlo Vancheri has received grants, speaker fees and/or advisory board fees from AstraZeneca, Boehringer Ingelheim, Chiesi, F. Hoffmann-La Roche, Ltd., Menarini and MSD.

Compliance with Ethics Guidelines. All patients gave written informed consent. The study conformed to the International Conference on Harmonisation (ICH) E6 and E2A guidelines for Good Clinical Practice and Clinical Safety Data Management, the principles of the Declaration of Helsinki, or the laws and regulations of the country where the research was conducted, whichever afforded the greater protection to the individual. The study was approved by the master ethics committee (Stichting BEBO, Postbus 1004, 9400 BA, Assen, The Netherlands) and by ethics committees at all other participating institutes.

Data Availability. Qualified researchers may request access to individual patient-level data through the clinical study data request platform (https://vivli.org/). Further details on Roche's criteria for eligible studies are available here (https://vivli.org/members/ourmembers/). For further details on Roche's Global Policy on the Sharing of Clinical Information and how to request access to related clinical study documents, see here (https://www.roche.com/ research_and_development/who_we_are_how_ we_work/clinical_trials/our_commitment_to_ data_sharing.htm).
Open Access. This article is licensed under a Creative Commons Attribution-NonCommercial 4.0 International License, which permits any non-commercial use, sharing, adaptation, distribution and reproduction in any medium or format, as long as you give appropriate credit to the original author(s) and the source, provide a link to the Creative Commons licence, and indicate if changes were made. The images or other third party material in this article are included in the article's Creative Commons licence, unless indicated otherwise in a credit line to the material. If material is not included in the article's Creative Commons licence and your intended use is not permitted by statutory regulation or exceeds the permitted use, you will need to obtain permission directly from the copyright holder. To view a copy of this licence, visit http:// creativecommons.org/licenses/by-nc/4.0/.

\section{REFERENCES}

1. Ryerson CJ, Collard HR. Update on the diagnosis and classification of ILD. Curr Opin Pulm Med. 2013;19:453-9.

2. Gibson GJ. Interstitial lung diseases. In: Gibson GJ, Loddenkemper R, Sibille Y, Lundbäck B, Fletcher M, editors. European Lung White Book. 2nd ed. Sheffield: European Respiratory Society; 2013. p. 256-69.

3. Cottin V, Hirani NA, Hotchkin DL, et al. Presentation, diagnosis and clinical course of the spectrum of progressive-fibrosing interstitial lung diseases. Eur Respir Rev. 2018;27:180076.

4. Richeldi L, du Bois RM, Raghu G, et al. Efficacy and safety of nintedanib in idiopathic pulmonary fibrosis. N Engl J Med. 2014;370:2071-82.

5. Noble PW, Albera C, Bradford WZ, et al. Pirfenidone in patients with idiopathic pulmonary fibrosis (CAPACITY): two randomised trials. Lancet. 2011;377:1760-9.

6. King TE Jr, Bradford WZ, Castro-Bernardini S, et al. A phase 3 trial of pirfenidone in patients with idiopathic pulmonary fibrosis. $\mathrm{N}$ Engl J Med. 2014;370:2083-92.

7. Fernandez Perez ER, Swigris JJ, Forssen AV, et al. Identifying an inciting antigen is associated with improved survival in patients with chronic hypersensitivity pneumonitis. Chest. 2013;144:1644-51. 
8. Distler O, Highland KB, Gahlemann M, et al. Nintedanib for systemic sclerosis-associated interstitial lung disease. N Engl J Med. 2019;380:2518-28.

9. Maher TM, Wuyts W. Management of fibrosing interstitial lung diseases. Adv Ther. 2019;36: 1518-31.

10. Russell AM, Ripamonti E, Vancheri C. Qualitative European survey of patients with idiopathic pulmonary fibrosis: patients' perspectives of the disease and treatment. BMC Pulm Med. 2016; 16:10.

11. Cosgrove GP, Bianchi P, Danese S, Lederer DJ. Barriers to timely diagnosis of interstitial lung disease in the real world: the INTENSITY survey. BMC Pulm Med. 2018;18:9.

12. Lamas DJ, Kawut SM, Bagiella E, Philip N, Arcasoy SM, Lederer DJ. Delayed access and survival in idiopathic pulmonary fibrosis: a cohort study. Am J Respir Crit Care Med. 2011;184:842-7.

13. Wells AU. Managing diagnostic procedures in idiopathic pulmonary fibrosis. Eur Respir Rev. 2013;22:158-62.

14. Bonella F, Wijsenbeek M, Molina-Molina M, et al. European IPF Patient Charter: unmet needs and a call to action for healthcare policymakers. Eur Respir J. 2016;47:597-606.

15. Hoyer N, Prior TS, Bendstrup E, Wilcke T, Shaker SB. Risk factors for diagnostic delay in idiopathic pulmonary fibrosis. Respir Res. 2019;20:103.

16. Lynch DA, Sverzellati N, Travis WD, et al. Diagnostic criteria for idiopathic pulmonary fibrosis: a Fleischner Society White Paper. Lancet Respir Med. 2018;6:138-53.

17. Wijsenbeek M, Bendstrup E, Valenzuela C, et al. Design of a study assessing disease behaviour during the peri-diagnostic period in patients with interstitial lung disease: the STARLINER Study. Adv Ther. 2019;36:232-43.

18. Maher TM, Corte TJ, Fischer A, et al. Pirfenidone in patients with unclassifiable progressive fibrosing interstitial lung disease: a double-blind, randomised, placebo-controlled, phase 2 trial. Lancet Respir Med. 2020;8:147-57.

19. Hegewald MJ, Gallo HM, Wilson EL. Accuracy and quality of spirometry in primary care offices. Ann Am Thorac Soc. 2016;13:2119-24.

20. Walters JA, Wood-Baker R, Walls J, Johns DP. Stability of the EasyOne ultrasonic spirometer for use in general practice. Respirology. 2006;11:306-10.
21. Nakshbandi G, Moor CC, Wijsenbeek MS. Home monitoring for patients with ILD and the COVID19 pandemic. Lancet Respir Med. 2020;8:1172-4.

22. Moor C, Mostard RLM, Grutters JC, et al. Home monitoring in patients with idiopathic pulmonary fibrosis: a randomized controlled trial. Am J Respir Crit Care Med. 2020;202:393-401.

23. Moor CC, van Manen MJG, Tak NC, van Noort E, Wijsenbeek MS. Development and feasibility of an eHealth tool for idiopathic pulmonary fibrosis. Eur Respir J. 2018;51:1702508.

24. Moor CC, Wapenaar M, Miedema JR, Geelhoed JJM, Chandoesing PP, Wijsenbeek MS. A home monitoring program including real-time wireless home spirometry in idiopathic pulmonary fibrosis: a pilot study on experiences and barriers. Respir Res. 2018;19:105.

25. Swigris S, Nathan S, Tighe R, et al. STARMAP: an observational study to assess disease-relevant outcomes using home-monitoring devices in patients with idiopathic pulmonary fibrosis (IPF). Eur Respir J. 2019;54(Suppl 63):PA1333.

26. Johannson KA, Vittinghoff E, Morisset J, Lee JS, Balmes JR, Collard HR. Home monitoring improves endpoint efficiency in idiopathic pulmonary fibrosis. Eur Respir J. 2017;50:1602406.

27. Wong AW, Fidler L, Marcoux V, et al. Practical considerations for the diagnosis and treatment of fibrotic interstitial lung disease during the coronavirus disease 2019 pandemic. Chest. 2020;158: 1069-78.

28. Khan F, Stewart I, Howard L, et al. The Its Not JUST Idiopathic pulmonary fibrosis Study (INJUSTIS): description of the protocol for a multicentre prospective observational cohort study identifying biomarkers of progressive fibrotic lung disease. BMJ Open Respir Res. 2019;6:e000439.

29. Tsai APY, Hur SA, Wong A, et al. Minimum important difference of the EQ-5D-5L and EQ-VAS in fibrotic interstitial lung disease. Thorax. 2020;76: 37-43.

30. Prior TS, Hoyer N, Hilberg O, Shaker SB, Davidsen JR, Bendstrup E. Responsiveness and minimal clinically important difference of SGRQ-I and K-BILD in idiopathic pulmonary fibrosis. Respir Res. 2020;21:91.

31. Travis WD, Costabel U, Hansell DM, et al. An official American Thoracic Society/European Respiratory Society statement: update of the international multidisciplinary classification of the idiopathic interstitial pneumonias. Am J Respir Crit Care Med. 2013;188:733-48. 